\title{
ГЛАДКОСТЬ РЕШЕНИЯ СЛАБО-СИНГУЛЯРНОГО ИНТЕГРАЛЬНОГО УРАВНЕНИЯ С РАЗРЫВНЫМ КОЭФФИЦИЕНТОМ
}

\author{
(Представил Г. Вайникко)
}

Рассматривается линейное интегральное уравнение второго рода

$$
u(t)=\int_{0}^{b} a(t, s) x(t-s) u(s) d s+f(t), \quad 0 \leqslant t \leqslant b,
$$

в котором $x \in C^{m-1}([-b, b] \backslash\{0\}), m \geqslant 1, \quad$ причем при $-b \leqslant t<0$ и $0<t \leqslant b$ справедлива оценка

$$
\left|x^{(m-1)}(t)\right| \leqslant c|t|^{-\beta}, \quad 0<\beta<m,
$$

где $\beta$ - дробное число.

В $\left[{ }^{1-7}\right]$ показано, что если $f \in C^{m}[0, b]$ и $a(t, s) \equiv 1$, то особенность функции $\boldsymbol{x}^{(k)}(t)$ влечет за собой особенности такого же порядка $(k+1)$-го производного решения в обоих концах отрезка интегрирования, а внутри промежутка $(0, b)$ решение $m$ раз непрерывно дифференцируемо. В случае достаточно гладкого по обоим переменным коэффициента $a(t, s)$ как это видно из $\left[{ }^{8-9}\right]$, характер особенностей производных решения останется таким же.

В настоящей работе изучается случай негладкого по параметру $s$ коэффициента $a(t, s)$. Отметим, что краткий исторический очерк и библиографические замечания к решению слабо-сингулярных интегральных уравнений даны в $\left[{ }^{9}\right]$.

1. Предположим, что $a(t, s) m$ раз непрерывно дифференцируема на $[0, b] \times[0, d]$ и $[0, b] \times[d, b]$ и целое $p(0 \leqslant p<\beta)$ - наименьшее, при котором $\frac{\partial^{p}}{\partial s^{p}} a(t, s)$ имеет при $s=d \quad(0<d<b)$ разрыв первого рода. Введем класс функций

$$
\begin{gathered}
E^{\beta}=\left\{u \in C[0, b] \cap C^{p}(0, b) \cap C^{m}((0, b) \backslash\{d\}):\right. \\
\left.\sup _{\substack{0<t<b \\
t \neq d}} \frac{\left|u^{(m)}(t)\right|}{t^{-\beta}+|t-d|^{-\beta+p}+(b-t)^{-\beta}}<\infty\right\} .
\end{gathered}
$$

Снабженное нормой

$$
\|u\|_{E^{\beta}}=\|u\|_{C}+\sup _{\substack{0<t<b \\ t \neq d}} \frac{\left|u^{(m)}(t)\right|}{t^{-\beta}+|t-d|^{-\beta+p}+(b-t)^{-\beta}},
$$

класс $E^{\beta}$ превращается в банахово пространство.

Справедливы следующие утверждения. 
Т е о рем а 1. Пусть $f \in E^{\beta}$ и пусть выполнены предположения о функциях $x(t)$ и $a(t, s)$. Если уравнение (1) имеет интегрируемое решение $u$, то $u \in E^{\beta}$.

Т е о р е м а 2. Пусть $f \in C^{m}[0, b]$, выполнены предположения о функциях $x(t)$ и $а(t, s)$ и, кроме того, в окрестности нуля справедливы оценки снизу

$$
\left|\chi^{(k)}(t)\right| \geqslant c_{k}|t|^{-v+m-1-k}, \quad 0<|t| \leqslant \delta, \quad k=0,1, \ldots, m-1,
$$

где $c_{k}=\mathrm{const}>0$, а ү удовлетворяет неравенствам

$$
\beta-(m-\beta)<\gamma \leqslant \beta .
$$

Тогда для тех $k=1, \ldots, m$, для которых $x^{(k-1)}(t)$ уже имеет при $t=0$ особенность, имеют место представления

$$
\begin{gathered}
u^{(k)}(t)=u(0) a(t, 0) x^{(k-1)}(t)-u(b) a(t, b) x^{(k-1)}(t-b)+v_{k}(t)+ \\
+(-1)^{p}\left(\begin{array}{c}
k-1 \\
p
\end{array}\right) u(d)\left[\left.\frac{\partial^{p}}{\partial s^{p}} a(t, s)\right|_{s=d+0}-.\right. \\
\left.-\left.\frac{\partial^{p}}{\partial s^{p}} a(t, s)\right|_{s=d-0}\right] x^{(k-1-p)}(t-d),
\end{gathered}
$$

где $v_{k} \in C^{m-k}((0, b) \backslash\{d\})$,

причем

$$
\lim _{t \rightarrow 0+} \frac{v_{k}(t)}{x^{(k-1)}(t)}=0, \quad \lim _{|t-d| \rightarrow 0} \frac{v_{k}(t)}{x^{(k-1-p)}(t-d)}=0, \quad \lim _{t \rightarrow b-} \frac{v_{k}(t)}{x^{(k-1)}(t-b)}=0 .
$$

В случае $k-1-p<0$ последнее слагаемое в выражении (5) отсутствует.

Теорема 2 говорит о том, что $(p+l)$-я $(1 \leqslant l \leqslant m-p)$ производная решения уравнения (1) при $t \rightarrow d-$ и $t \rightarrow d+$ имеет такие же особенности, как функция $x^{(l-1)}(t)$ соответственно при $t \rightarrow 0-$ и $t \rightarrow 0+$. Доказательство этой теоремы построено в пункте 5 .

Доказательство теоремы 1 основывается на следующем результате (см. $\left[{ }^{9}\right]$, с. 10$)$ :

Пусть $E$ и $E_{1}$ - банаховы пространства, причем $E$ непрерывно и плотно вложено в $E_{1}$. Пусть $T: E \rightarrow E$ и $K: E_{1} \rightarrow E_{1}-$ линейные вполне непрерывные операторы, причем $T \subset K$ является сужением оператора $K$ на $E$. Если уравнение $u_{1}+K u_{1}=f$ с $f \in E \subset E_{1}$ разрешимо в $E_{1}$, то его решения принадлежат $E$.

Положим $E=E^{\beta}$ и $E_{1}=L_{1}=L_{1}[0, b]$,

$$
(T u)(t)=\int_{0}^{b} a(t, s) x(t-s) u(s) d s, \quad u \in E^{\beta}
$$

и

$$
(K u)(t)=\int_{0}^{b} a(t, s) \chi(t-s) u(s) d s, \quad u \in L_{1} .
$$

Полная непрерывность оператора $K: L_{1} \rightarrow L_{1}$ в условиях (2) очевидна, доказательство полной непрерывности оператора $T$ в $E^{\beta}$ вынесено в пунктах $2-4$. Применение этого результата дает, что в случае $f \in E^{\beta}$ решение уравнения (1) принадлежит $E^{\beta}$.

2. Заметим, что из (2) вытекают неравенства

$$
\left|x^{(k)}(t)\right| \leqslant c_{k}\left(|t|^{-\beta+m-1-k}+1\right), \quad k=0,1, \ldots, m-1,
$$

которые в дальнейшем будут неоднократно использованы, 
Введем следующие обозначения

$$
\begin{gathered}
a_{0}(t, s)=a(t, s)= \begin{cases}a_{0}^{-}(t, s), & 0 \leqslant s \leqslant d, \\
a_{0}^{+}(t, s), & d \leqslant s \leqslant b,\end{cases} \\
a_{i}(t, s)=\left(\frac{\partial}{\partial t}+\frac{\partial}{\partial s}\right) a_{i-1}(t, s)= \begin{cases}a_{i}^{-}(t, s), & 0 \leqslant s \leqslant d, \\
a_{i}^{+}(t, s), & d \leqslant s \leqslant b,\end{cases}
\end{gathered}
$$

и интегральные операторы

$$
\begin{aligned}
\left(T_{i} u\right)(t)= & \int_{0}^{b} a_{i}(t, s) \varkappa(t-s) u(s) d s=\int_{0}^{d} a_{i}^{-}(t, s) \varkappa(t-s) u(s) d s+ \\
& +\int_{d}^{b} a_{i}^{+}(t, s) x(t-s) u(s) d s, \quad i=1, \ldots, m .
\end{aligned}
$$

Ясно, что сопряженный к $T_{i}$ оператор $T_{i}{ }^{*}$ выражается в виде

$$
\left(T_{\imath}^{*} u\right)(s)=\int_{0}^{b} a_{i}(t, s) x(t-s) u(t) d t= \begin{cases}\int_{0}^{b} a_{i}^{-}(t, s) x(t-s) u(t) d t, \quad 0 \leqslant s \leqslant d, \\ \int_{0}^{b} a_{i}^{+}(t, s) x(t-s) u(t) d t, \quad d \leqslant s \leqslant b .\end{cases}
$$

Предложени е 1 . Для $u \in E^{\beta}$ справедлива формула

$$
\begin{gathered}
(T u)^{\prime}(t)=\left(T u^{\prime}\right)(t)+\left(T_{1} u\right)(t)+u(0) a(t, 0) x(t)-u(b) a(t, b) x(t-b)+ \\
+u(d)\left[a_{0}^{+}(t, d)-a_{0}^{-}(t, d)\right] x(t-d) .
\end{gathered}
$$

Доказ ательство. Заменой $t-s=\sigma$ запишем $T u$ в виде

$$
\begin{aligned}
(T u)(t)= & -\int_{t}^{t-d} a_{0}^{-}(t, t-\sigma) x(\sigma) u(t-\sigma) d \sigma- \\
& -\int_{t-d}^{t-b} a_{0}^{+}(t, t-\sigma) x(\sigma) u(t-\sigma) d \sigma .
\end{aligned}
$$

Известное правило дифференцирования таких интегралов дает

$$
\begin{gathered}
(T u)^{\prime}(t)=-\int^{t-d}\left[a_{1}^{-}(t, t-\sigma) x(\sigma) u(t-\sigma)+\right. \\
\left.+a_{0}^{-}(t, t-\sigma) x(\sigma) u^{\prime}(t-\sigma)\right] d \sigma-\left[a_{0}^{-}(t, t-\sigma) x(\sigma) u(t-\sigma)\right]_{\sigma=t}^{\sigma=t-d}- \\
-\left[a_{0}^{+}(t, t-\sigma) x(\sigma) u(t-\sigma)\right]_{\sigma=t-d}^{\sigma=t-b}-\int_{t-d}^{t-b}\left[a_{1}^{+}(t, t-\sigma) x(\sigma) u(t-\sigma)+\right. \\
\left.+a_{0}^{+}(t, t-\sigma) \varkappa(\sigma) u^{\prime}(t-\sigma)\right] d \sigma .
\end{gathered}
$$

Вернувшись в подынтегральных членах к переменной $s$, после несложных преобразований приходим к формуле (8).

Предложение 1 доказано.

В ходе рассуждений нам понадобится понятие обобщенных производных (см. напр., $\left.\left[{ }^{10}\right]\right)$. Обозначим через $\langle z, \varphi\rangle$ значение обобщенной функции $z$ на бесконечно дифференцируемой финитной в $\Omega_{d}=$ $=\{(0, b) \backslash\{d\}\}$ функции $\varphi$ (основные функции). Отметим, что если $m$-я обобщенная производная $D^{m} z \in C(0, b)$, то $z$ дифференцируема и в 
классическом смысле, и обобщенная производная совпадает с классической: $D^{m z}=z^{(m)}$. Для основных функций $\varphi$ справедливы следующие равенства (см. $\left[{ }^{9}\right]$, с. 13$)$ :

$$
T_{0}^{*} D^{k} \varphi=\sum_{i=0}^{k}(-1)^{k-i}\left(\begin{array}{c}
k \\
i
\end{array}\right) D^{i} T_{k-i}^{*} \varphi, \quad k=0, \ldots, m .
$$

При выводе формулы для высших производных от $T u$ будем предполагать, что $u \in E^{\beta}$ имеет особенности только в точке $d \in(0, b)$.

Пр едложени е 2. Пусть $m \geqslant 2$,

$$
u \in C^{p}[0, b] \cap C^{m}([0, b] \backslash\{d\}), \quad u^{(i)}(0)=u^{(i)}(b)=0,
$$

$i=0,1, \ldots, m, \quad\left|u^{(m)}(t)\right| \leqslant c\left(|t-d|^{-\beta+p}+1\right), \quad t \in\{[0, b] \backslash\{d\}\}$,

$$
0<\beta<m \text {. }
$$

Тогда для $k=2, \ldots$, , справедлива формула

$$
\begin{gathered}
\left(D^{k} T u\right)(t)=\sum_{i=0}^{k}\left(\begin{array}{l}
k \\
i
\end{array}\right)\left\{\int_{0}^{d} g_{i, k}^{-}(t, s) u^{(i)}(s) d s+\int_{d}^{b} g_{i, k}^{+}(t, s) u^{(i)}(s) d s\right\}+ \\
+(-1)^{k-1} u(d)\left\{\left[\frac{\partial^{k-1}}{\partial s^{k-1}}\left(a_{0}^{+}(t, s) x(t-s)\right)\right]_{s=d}-\right. \\
\left.-\left[\frac{\partial^{k-1}}{\partial s^{k-1}}\left(a_{0}^{-}(t, s) \mid x(t-s)\right)\right]_{s=d}\right\},
\end{gathered}
$$

гेe

$$
\begin{aligned}
& g_{0, k}^{ \pm}(t, s)=a_{k}^{ \pm}(t, s) x(t-s), \\
& g_{i, k}^{ \pm}(t, s)=a_{k-i}^{ \pm}(t, s) x(t-s)-\sum_{j=0}^{i-1} x_{k-i, j}^{ \pm}(t) \frac{(s-d)^{j}}{j !}, \\
& g_{k, k}^{ \pm}(t, s)=a_{0}^{ \pm}(t, s) x(t-s)-\sum_{j=0}^{k-2} x_{0, j}^{ \pm}(t) \frac{(s-d)^{j}}{j !}, k-1, \\
& x_{i, j}^{ \pm}(t)=\left[\frac{\partial^{j}\left(a_{i}^{ \pm}(t, s) x(t-s)\right)}{\partial s^{j}}\right]_{s=d}, \quad t \neq d, \quad i+j \leqslant m .
\end{aligned}
$$

Д оказ а тельство. Для любой основной функции $\varphi$ в силу (9) имеем

$$
\begin{gathered}
\left\langle D^{k} T u, \varphi\right\rangle=(-1)^{k}\left\langle T u, D^{k} \varphi\right\rangle=(-1)^{k}\left\langle u, T^{*} D^{k} \varphi\right\rangle= \\
=(-1)^{k} \int_{0}^{b} u(s)\left(T^{*} D^{k} \varphi\right)(s) d s= \\
=(-1)^{k} \int_{0}^{b} u(s)\left[\sum_{i=0}^{k}(-1)^{k-i}\left(\begin{array}{c}
k \\
i
\end{array}\right)\left(D^{i} T_{k-i}^{*} \varphi\right)(s)\right] d s=\int_{0}^{b} u(s)\left(T_{k}^{*} \varphi\right)(s) d s+ \\
+\sum_{i=1}^{k}(-1)^{i}\left(\begin{array}{c}
k \\
i
\end{array}\right) \int_{0}^{d} u(s) \frac{d^{i}}{d s^{i}}\left[\left(T_{k-i}^{*} \varphi\right)(s)-v_{i, k}^{-}(s)\right] d s+ \\
+\sum_{i=1}^{k}(-1)^{i}\left(\begin{array}{c}
k \\
i
\end{array}\right) \int_{d}^{b} u(s) \frac{d^{i}}{d s^{i}}\left[\left(T_{k-i}^{*} \varphi\right)(s)-v_{i, k}^{+}(s)\right] d s,
\end{gathered}
$$


где $v_{i, k}^{ \pm}(s)$ - произвольные многочлены степени $i-1$. Мы их выбираем так:

$$
\begin{aligned}
& v_{i, k}^{ \pm}(s)=\sum_{j=0}^{i-1}\left[\frac{d^{j}}{d s^{j}}\left(T_{k-i}^{*} \mid \varphi\right)(s)\right]_{s=d \pm} \cdot \frac{(s-d)^{j}}{j !}, \quad i=1, \ldots, k-1, \\
& v_{k, k}^{ \pm}(s)=\sum_{j=0}^{k-2}\left[\frac{d^{j}}{d s^{j}}\left(T_{0}^{*} \varphi\right)(s)\right]_{s=d \pm} \cdot \frac{(s-d)^{j}}{j !} .
\end{aligned}
$$

Тогда для $i=1,2, \ldots, k-1$ функции $\quad\left(T_{k-i}^{*} \varphi\right)(s)-v_{i, k}^{ \pm}(s) \quad$ и их производные до порядка $i-1$ включительно обращаются в точке $s=d$ в нуль; при $i=k$ аннулируются производные до порядка $k-2$. Учитывая (10), интегрированием по частям находим

$$
\begin{aligned}
& \left\langle D^{k} T u, \varphi\right\rangle=\int_{0}^{b} u(s)\left(T_{k}^{*} \varphi\right)(s) d s+ \\
& +\sum_{i=1}^{k}\left(\begin{array}{c}
k \\
i
\end{array}\right) \int_{0}^{d} u^{(i)}(s)\left[\left(T_{k-1}^{*} \varphi\right)(s)-v_{i, k}^{-}(s)\right] d s+ \\
& +\sum_{i=1}^{k}\left(\begin{array}{c}
k \\
i
\end{array}\right) \int_{d}^{b} u^{(i)}(s)\left[\left(T_{k-i}^{*} \varphi\right)(s)-v_{i, k}^{+}(s)\right] d s+ \\
& +(-1)^{k}\left\{u(s) \frac{d^{k-1}}{d s^{k-1}}\left[\left(T_{0}^{*} \varphi\right)(s)\right]\right\}_{s=0}^{s=d-}+ \\
& +(-1)^{k}\left\{u(s) \frac{d^{k-1}}{d s^{k-1}}\left[\left(T_{0}^{*} \varphi\right)(s)\right]\right\}_{s=d+}^{s=b},
\end{aligned}
$$

остальные внеинтегральные члены аннулируются. В интегралах

$$
\left(T_{i}^{*} \varphi\right)(s)= \begin{cases}\int_{0}^{b} a_{i}^{-}(t, s) \varkappa(t-s) \varphi(t) d t, & 0 \leqslant s \leqslant d, \\ \int_{0}^{b} a_{i}^{+}(t, s) x(t-s) \varphi(t) d t, & d \leqslant s \leqslant b\end{cases}
$$

интегрирование в действительности происходит по носителю supp $\varphi \subset \Omega_{d}$ и при $s=d$ аргумент $t-s$ функции $x(t-s)$ отделен от нуля. Это позволяет вычислять производные при $s=d$ дифференцированием под знаком интеграла:

$$
\begin{aligned}
\left.\frac{d^{j}}{d s^{j}}\left(T_{i}^{*} \varphi\right)(s)\right|_{s=d \pm}= & \int_{0}^{b}\left[\frac{\partial^{j}}{\partial s^{j}}\left(a_{i}^{ \pm}(t, s) x(t-s)\right)\right]_{s=d} \varphi(t) d t= \\
& =\int_{0}^{b} x_{i, j}^{ \pm}(t) \varphi(t) d t .
\end{aligned}
$$

Проделав соответствующие подстановки, имеем

$$
\left\langle D^{k} T u, \varphi\right\rangle=\int_{0}^{d} u(s) \int_{0}^{b} a_{k}^{-}(t, s) x(t-s) \varphi(t) d t d s+
$$




$$
\begin{aligned}
& +\int_{d}^{b} u(s) \int_{0}^{b} a_{k}^{+}(t, s) x(t-s) \varphi(t) d t d s+ \\
& +\sum_{i=1}^{k-1}\left(\begin{array}{c}
k \\
i
\end{array}\right) \int_{0}^{d} u^{(i)}(s)\left\{\int_{0}^{b} a_{k-i}^{-}(t, s) x(t-s) \varphi(t) d t-\right. \\
& \left.-\sum_{j=0}^{i-1} \int_{0}^{b} x_{i, j}^{-}(t) \varphi(t) d t \frac{(s-d)^{j}}{j !}\right\} d s+ \\
& +\int_{0}^{d} u^{(k)}(s)\left\{\int_{0}^{b}\left[a_{0}^{-}(t, s) x(t-s)-\sum_{j=0}^{k-2} x_{0, j}^{-}(t), \frac{(s-d)^{j}}{j !}\right] \varphi(t) d t\right\} d s+ \\
& +\sum_{i=1}^{k-1}\left(\begin{array}{l}
k \\
i
\end{array}\right) \int_{d}^{b} u^{(i)}(s)\left\{\int_{0}^{b} a_{k-i}^{+}(t, s) x(t-s) \varphi(t) d t-\right. \\
& \left.-\sum_{j=0}^{i-1} \int_{0}^{b} x_{i, j}^{+}(t) \varphi(t) d t \frac{(s-d)^{j}}{j !}\right\} d s+ \\
& +\int_{d}^{b} u^{(k)}(s)\left\{\int_{0}^{b}\left[\mathrm{a}_{0}^{+}(t, s) x(t-s)-\sum_{j=0}^{k-2} x_{0, j}^{+}(t) ! \frac{\left(s-d 2^{j}\right.}{j !}\right] \varphi(t) d t\right\} d s+ \\
& +(-1)^{k} u(d) \int_{0}^{b}\left[x_{0, k-1}^{-}(t)-x_{0, k-1}^{+}(t)\right] \varphi(t) d t .
\end{aligned}
$$

В обозначениях (12) это можно записать в виде

$$
\begin{aligned}
\left\langle D^{k} T u, \varphi\right\rangle= & (-1)^{k} u(d) \int_{0}^{b}\left[x_{0, k-1}^{-}(t)-x_{0, k-1}^{+}(t)\right] \varphi(t) d t+ \\
& +\sum_{i=0}^{k}\left(\begin{array}{c}
k \\
i
\end{array}\right) \int_{0}^{d}\left\{\int_{0}^{b} g_{i, k}^{-}(t, s) \varphi(t) d t\right\} u^{(i)}(s) d s+ \\
& +\sum_{i=0}^{k}\left(\begin{array}{c}
k \\
i
\end{array}\right) \int_{d}^{b}\left\{\int_{0}^{b} g_{i, k}^{+}(t, s) \varphi(t) d t\right\} u^{(i)}(s) d s .
\end{aligned}
$$

Установим правомерность изменения в последних интегралах порядка интегрирования. Достаточно показать, что сходятся двойные интегралы

$\int_{0}^{b} \int_{0}^{d}\left|u^{(i)}(s)\right|\left|g_{i, k}^{-}(t, s)\right||\varphi(t)| d s d t \quad$ и $\quad \int_{0}^{b} \int_{d}^{b}\left|u^{(i)}(s)\right|\left|g_{i, k}^{+}(t, s)\right||\varphi(t)| d s d t$ или любой из соответствующих повторных интегралов. Из (10) следуют оценки

$$
\left|u^{(i)}(s)\right| \leqslant c_{i}\left(|s-d|^{-\beta+m-i+p}+1\right), \quad i=0,1 \ldots, m,
$$


а из (2) и (7), что

$$
\begin{aligned}
& \left|g_{i, 0}^{ \pm}(t, s)\right| \leqslant c|t-s|^{-\beta+m-1} \\
& \left|g_{i, k}^{ \pm}(t, s)\right| \leqslant c\left(|t-s|^{-\beta+m-1}+\sum_{j=0}^{i-1}\left(|t-d|^{-\beta+m-j-1}+1\right)(s-d)^{j}\right), \\
& \mid g_{k, k}^{ \pm}(t, s) \leqslant c\left(|t-s|^{-\beta+m-1}+\sum_{j=0}^{k-2}\left(|t-d|^{-\beta+m-j-1}+1\right)(s-d)^{j}\right) .
\end{aligned}
$$

Но эти оценки слишком грубы около $s=d$. Трактуя $g_{i, k}^{ \pm}(t, s)$ как остаточные члены формулы Тейлора для функций $a_{k-i}^{ \pm}(t, s) x(t-s)$, можем оценить

$$
\begin{aligned}
& \left|g_{i, k}^{-}(t, s)\right| \leqslant \max _{s \leqslant \rho \leqslant d} \frac{\partial^{i}}{\partial \varrho^{i}}\left[a_{k-i}^{-}(t, \varrho) x(t-\varrho)\right] \frac{(s-d)^{i}}{i !}, \\
& \left|g_{i, k}^{+}(t, s)\right| \leqslant \max _{d \leqslant \rho \leqslant s} \frac{\partial^{i}}{\partial \varrho^{i}}\left[a_{k-i}^{+}(t, \varrho) x(t-\varrho)\right] \frac{(s-d)^{i}}{i !},
\end{aligned}
$$

откуда для близких к $d$ переменных $s(|s-d| \leqslant|t-d| / 2)$ получаем $\left|g_{i, k}^{ \pm}(t, s)\right| \leqslant c\left(|t-d|^{-\beta+m-1-i}+1\right)(s-d)^{i}, \quad i=0,1, \ldots, k-1$,

$\left|g_{k, k}^{ \pm}(t, s)\right| \leqslant c\left(|t-d|^{-\beta+m-k}+1\right)(s-d)^{k}$.

\section{В интеграле}

$$
\int_{0}^{d}\left|u^{(i)}(s)\right|\left|g_{i, k}^{-}(t, s)\right| d s \leqslant c_{i}\left(\int_{0}^{\frac{t+d}{2}}+\int_{\frac{t+d}{2}}^{d}\right) \times
$$

$$
\times\left(\left|g_{i, k}^{-}(t, s)\right|\left(|s-d|^{-\beta+m-i+p}+1\right) d s\right), \quad i=0,1, \ldots, k
$$

для $(t+d) / 2 \leqslant s \leqslant d$ воспользуемся оценкой (16) или (17), а для $0<s<(t+d) / 2$ оценкой (15). Аналогично поступаем с интегралом

$$
\int_{d}^{b}\left|u^{(i)}(s)\right|\left|g_{i, k}^{+}(t, s)\right| d s .
$$

Несложный подсчет показывает, что

$$
\begin{aligned}
& \int_{0}^{d}\left|u^{(i)}(s)\right|\left|g_{i, k}^{-}(t, s)\right| d s \leqslant c\left(|t-d|^{2(m-\beta)-i+p}+1\right), \\
& \int_{d}^{b}\left|u^{(i)}(s)\right|\left|g_{i, k}^{+}(t, s)\right| d s \leqslant c\left(|t-d|^{2(m-\beta)-i+p}+1\right),
\end{aligned}
$$

Теперь, учитывая финитность функции $\varphi$, заключаем, что повторные интегралы

$$
\int_{0}^{b}\left\{\int_{0}^{d}\left|u^{(i)}(s)\right|\left|g_{i, k}^{-}(t, s)\right| d s\right\} \varphi(t) d t \quad \text { и } \quad \int_{0}^{b}\left\{\int_{d}^{b}\left|u^{(i)}(s)\right|\left|g_{i, k}^{+}(t, s)\right| d s\right\} \varphi(t) d t
$$

сходятся.

Поменяв порядок интегрирования в (13), имеем 


$$
\begin{gathered}
\left\langle D^{k} T u, \varphi\right\rangle=(-1)^{k-1} u(d) \int_{0}^{b}\left[x_{0, k-1}^{+}(t)-x_{0, k-1}^{-}(t)\right] \varphi(t) d t+ \\
+\int_{0}^{b} \sum_{i=0}^{k}\left(\begin{array}{c}
k \\
i
\end{array}\right)\left\{\int_{0}^{d} g_{i k}^{-}(t, s) u^{(i)}(s) d s+\int_{d}^{b} g_{i, k}^{+}(t, s) u^{(i)}(s) d s\right\} \varphi(t) d t
\end{gathered}
$$

откуда, ввиду произвольности $\varphi$, вытекает утверждение предложения 2 .

Из проведенных оценок следует также сходимость интегралов в (11) и неравенство

$$
\begin{gathered}
\left|\left(D^{k} T u\right)(t)-(-1)^{k-1} u(d)\left[x_{0, k-1}^{+}(t)-x_{0, k-1}^{-}(t)\right]\right| \leqslant \\
\leqslant c\left(|t-d|^{2(m-\beta)-k+p}+1\right), \quad 2 \leqslant k \leqslant m .
\end{gathered}
$$

Функции $g_{i, k}^{ \pm}(t, s)$ непрерывны при $t \neq s, t \neq d$, а особенность при $t=s-$ суммируемая. Легко видно, что интегралы в (11) представляют собой непрерывные при $t \neq d$ функции. Таким образом, производные $D^{k} T u$ в (11) в действительности классические.

3. Обозначим через $E_{d}{ }^{\beta}$ подпространство банахова пространства $E^{\beta}$, состоящего из удовлетворяющих условиям (10) функций.

Пр едло жен и е 3. Оператор $T$ переводит $E_{d}{ }^{\beta}$ в $E^{\beta}$ и вполне непрерывен между этими пространствами.

Д ок а з а т е л с т в о. Известно, что слабо-сингулярный интегральный оператор действует и вполне непрерывен в $C[0, b]$. В предложении 2 установлено, что для $u \in E_{d}^{\beta}$ существует производная $\frac{d^{m}}{d t^{m}}(T u)(t) \quad$ в $(0, d)$ и в $(d, b)$. В силу $(18)$

$$
\begin{aligned}
\mid \frac{d^{m}}{d t^{m}}(T u)(t)- & (-1)^{m-1} u(d)\left[\chi_{0, m-1}^{+}(t)-\chi_{0, m-1}^{-}(t)\right] \mid \leqslant \\
& \leqslant c\left(|t-d|^{(m-\beta)-\beta+p}+1\right),
\end{aligned}
$$

а в силу $(7),(12)$ и предположения о гладкости функции $a(t, s)$

$$
\begin{gathered}
\left|x_{0, m-1}^{+}(t)-x_{0, m-1}^{-}(t)\right|=\mid \sum_{i=0}^{m-1}\left(\begin{array}{c}
m-1 \\
i
\end{array}\right)\left\{\left.\frac{\partial^{i}}{\partial s^{i}} a^{+}(t, s)\right|_{s=d} \times\right. \\
\left.\times\left.\frac{\partial^{m-1-i}}{d s^{m-1-i}} x(t-s)\right|_{s=d}-\left.\frac{\partial^{i}}{\partial s^{i}} a^{-}(t, s)\right|_{s=d} \times\left.\frac{\partial^{m-1-1}}{\partial s^{m-1-i}} x(t-s)\right|_{s=d}\right\} \mid \leqslant \\
\leqslant\left|\sum_{i=p}^{m-1} c \frac{\partial^{m-1-i}}{\partial s^{m-1-i}} x(t-s)\right|_{s=d} \mid \leqslant c_{1}\left(|t-d|^{-\beta+p}+1\right) .
\end{gathered}
$$

Значит, $T$ переводит $E_{d}^{\beta}$ в $E^{\beta}$.

Докажем полную непрерывность $T: E_{d}^{\beta} \rightarrow E^{\beta}$. Пусть $\left\{u_{n}\right\}-$ ограниченная в $E_{d}^{\beta}$ последовательность. Это равносильно неравенствам (14) для $u_{n}$ с независящими от $n$ постоянными $c_{i}$. Для доказательства компактности $\left\{T u_{n}\right\}$ в $E^{\beta}$, нам удобно установить компактность последовательности $\left\{T u_{n}+z_{n}\right\}$ в $E^{\beta}$, где $\left\{z_{n}\right\}$ с

$$
\begin{aligned}
& z_{n}(t)=(-1)^{m} u_{n}(d) \int^{t} d t \ldots \int^{t}\left[x_{0, m-1}^{+}(t)-x_{0, m-1}^{-}(t)\right] d t
\end{aligned}
$$

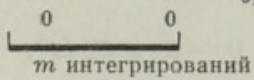

- компактная последовательность в $E^{\beta}$. Отметим, что $z_{n}^{(m)}(t)=$ $=(-1)^{m} u_{n}(d)\left[x_{0, m-1}^{+}(t)-x_{0, m-1}^{-}(t)\right] . \quad$ Для установления компактности 
некой последовательности $\left\{y_{n}\right\}$ в $E^{\beta}$ достаточно показать, что последовательности $\left\{y_{n}\right\}$ и $\left\{\left[t^{\beta}+|t-d|^{\beta-p}+(b-t)^{\beta}\right] y_{n}^{(m)}(t)\right\}$ компактны в $C[0, b]$. Поскольку последовательность $\left\{T u_{n}+z_{n}\right\}$ компактна в $C[0, b]$, то дело сводится к доказательству компактности последовательности $\left\{|t-d|^{\beta-p} \frac{d^{m}}{d t^{m}}\left(T u_{n}+z_{n}\right)\right\}_{3} C[0, b]$. (Учитывая (18) и (19), видно, что в оценках для $\frac{d^{m}}{d t^{m}}\left(T u_{n}+z_{n}\right)$ доминирует только величина $|t-d|$ и мы можем $t^{\beta}$ и $(b-t)^{\beta}$ опустить.) Последнее, в свою очередь, можно с учетом (11) свести к установлению полной непрерывности интегральных операторов $G_{i}^{-}: L_{\infty}[0, d] \rightarrow C[0, d]$ и $G_{i}^{++}: L_{\infty}[d, b] \rightarrow C[d, b]$, где

$$
\begin{gathered}
\left(G_{i}^{-} v\right)(t)=\int_{0}^{d} G_{i}^{-}(t, s) v(s) d s \quad \text { и } \quad\left(G_{i}^{+} v\right)(t)=\int_{d}^{b} G_{i}^{+}(t, s) v(s) d s, \\
G_{i}^{ \pm}(t, s)=|t-d|^{\beta-p} g_{i, m}^{ \pm}(t, s)\left(|s-d|^{-\beta+p+m-i}+1\right), \quad i=0,1, \ldots, m .
\end{gathered}
$$

Ядра $G_{i}^{ \pm}(t, s)$ имеют особенности при $s=d$ и $s=t$. Из оценок (15), (16) и (17) вытекает, что

$$
\begin{gathered}
\left|G_{i}^{ \pm}(t, s)\right| \leqslant \\
\leqslant c\left\{\begin{array}{lll}
\left(|s-d|^{-\beta+m-1}+|t-s|^{-\beta+m-1}+1\right) & \text { при } & |s-d| \leqslant|t-d| / 2, \\
\left(|t-s|^{-\beta+m-1}+1\right. & \text { при } & |s-d|>|t-d| / 2,
\end{array}\right.
\end{gathered}
$$

$i=0,1, \ldots, m$. (Мы учли, что $|t-d|>0$, во втором случае.) Поскольку $-\beta+m-1>-1$, особенности $G_{i}^{ \pm}(t, s)$ слабые и полная непрерывность $G_{i}: L_{\infty}[0, b] \rightarrow C[0, b]$ устанавливается стандартным образом подавлением особенностей (см. напр. $\left[{ }^{9}\right]$, с. 19).

Предложение 3 доказано.

4. Пр едло же н и е 4. Интегральный оператор $T$ переводит пространство $E^{\beta}$ в себя и вполне непрерывен в этом пространстве.

Д ок а з а тельство. Кроме $E_{d}^{\beta}$ введем еще подпространства $E_{0}{ }^{\beta} \subset E^{\beta}$ и $E_{b}^{\beta} \subset E^{\beta}$, состоящие из функций, удовлетворяющих условиям

$$
\begin{gathered}
u \in C[0, b] \cap C^{m}(0, b], \quad u^{(i)}(d)=u^{(i)}(b)=0, \quad i=0,1, \ldots, m, \\
\left|u^{(m)}(t)\right| \leqslant c t^{-\beta} \quad \text { в } E_{0}^{\beta} \text { и } \\
u \in C[0, b] \cap C^{m}[0, b), \quad u^{(i)}(0)=u^{(i)}(d)=0, \quad i=0,1, \ldots, m, \\
\mid u^{(m)}(t) \leqslant c(b-t)^{-\beta} \text { в } E_{b}^{\beta} .
\end{gathered}
$$

Проводя аналогичные пунктам $2-3$ рассуждения (см. также $\left.\left[{ }^{9}\right], \S 1\right)$, ясно, что $T$ переводит $E_{0}^{\beta}$ в $E^{\beta}$ и $E_{b}^{\beta}$ в $E^{\beta}$ и вполне непрерывен между этими пространствами.

Зафиксируем две какие-либо функции $e, h \in C^{\infty}[0, b]$ такие, что $e(t)=1$ для $t \in[0, d / 3]$ и $e(t)=0$ для $t \in[2 d / 3, b]$, а $h(t)=0$ для $t \in[0, b-2(b-d) / 3$ и $h(t)=1$ для $t \in[b-(b-d) / 3, b]$. Любую функцию $u \in E^{\beta}$ представим в виде

$$
\begin{gathered}
u=v+w+z, \quad v=e \cdot u \in E_{0}^{\beta}, \quad z=h \cdot u \in E_{b}^{\beta}, \\
w=(1-e-h) \cdot u \in E_{d}^{\beta} .
\end{gathered}
$$

Тогда $T u=T v+T w+T z \in E^{\beta}, \quad$ т. е. оператор $T$ переводит $E^{\beta}$ в себя. 
Если $\left\{u_{n}\right\}$ - ограниченная в $E^{\beta}$ последовательность, то по формулам (20) полученные последовательности $\left\{v_{n}\right\},\left\{w_{n}\right\},\left\{z_{n}\right\}$ ограниченные, соответственно, в $E_{0}{ }^{\beta}, E_{d}^{\beta}$ и $E_{b}^{\beta}$. Следовательно, $\left\{T u_{n}\right\},\left\{T w_{n}\right\}$ и $\left\{T z_{n}\right\}$ компактны в $E^{\beta}$. Это доказывает полную непрерывность оператора $T$ в $E^{\beta}$.

Отметим, что аналогично (18) для функций $v \in E_{0}^{\beta}$ и $z \in E_{b}{ }^{\beta}$ справедливы неравенства

$$
\begin{aligned}
& \left|\left(D^{k} T v\right)(t)-(-1)^{k-1} v(0) \bar{\varkappa}_{0, k-1}(t)\right| \leqslant c\left(t^{2(m-\beta)-k}+1\right), \\
& \left|\left(D^{k} T z\right)(t)-(-1)^{k} z(b) \tilde{\chi}_{0, k-1}(t)\right| \leqslant c\left((b-t)^{2(m-\beta)-k}+1\right),
\end{aligned}
$$

где

$$
\begin{aligned}
& \bar{x}_{0, k-1}(t)=\frac{\partial^{k-1}}{\partial s^{k-1}}\left[a_{0}^{-}(t, s) x(t-s)\right]_{s=0}, \\
& \tilde{x}_{0, k-1}(t)=\frac{\partial^{k-1}}{\partial s^{k-1}}\left[a_{0}^{+}(t, s) x(t-s)\right]_{s=b}, \quad k=2, \ldots, m .
\end{aligned}
$$

5. Доказательство теоремы 2. Пусть $f \in E^{\beta}$ (позже это условие усилим), а $u(t)-$ решение уравнения (1). По теореме $1 u(t) \in E^{\beta}$ и дифференцированием (1) находим

$$
u^{(k)}(t)=\frac{d^{k}}{d t^{k}}(T u)(t)+f^{(k)}(t), \quad 0<t<b, \quad t \neq d, \quad k=2, \ldots, m .
$$

Представив $u(t)$ в виде $(20)$, получим

$$
u^{(k)}(t)=\frac{d^{k}}{d t^{k}}(T v)(t)+\frac{d^{k}}{d t^{k}}(T w)(t)+\frac{d^{k}}{d t^{k}}(T z)(t)+f^{(k)}(t) .
$$

Из неравенства (18) для $ш \in E_{d}^{\beta}$ и (21) имеем

$$
\begin{aligned}
\frac{d^{k}}{d t^{k}}(T v)(t)= & (-1)^{k-1} v(0) \bar{x}_{0, k-1}(t)+O\left(t^{2(m-\beta)-k}+1\right), \\
\frac{d^{k}}{d t^{k}}(T w)(t)= & (-1)^{k-1} w(d)\left[\chi_{0, k-1}^{+}(t)-x_{0, k-1}^{-}(t)\right]+ \\
& \quad+O\left(|t-d|^{2(m-\beta)-k+p}+1\right), \\
\frac{d^{k}}{d t^{k}}(T z)(t)= & (-1)^{k} z(b) \tilde{\chi}_{0, k-1}(t)+O\left((b-t)^{2(m-\beta)-k}+1\right),
\end{aligned}
$$

где $v(0)=u(0)$, $w(d)=u(d)$ и $z(b)=u(b)$. В силу предположения гладкости функции $a(t, s)$ получим (см. также (19))

$$
\begin{gathered}
x_{0, k-1}^{+}(t)-x_{0, k-1}^{-}(t)=\frac{\partial^{k-1}}{\partial s^{k-1}}\left[\left(a_{0}^{+}(t, s)-a_{0}^{-}(t, s)\right) x(t-s)\right]_{s=d}= \\
=\left.(-1)^{k-1-p}\left(\begin{array}{c}
k-1 \\
p
\end{array}\right) \frac{\partial^{p}}{\partial s^{p}}\left[a_{0}^{+}(t, s)-a_{0}^{-}(t, s)\right] \frac{\partial s^{k-1-p}}{\partial s^{k-1-p}} x(t-s)\right|_{s=d}+ \\
+O\left(x^{(k-2-p)}(t-d)\right)
\end{gathered}
$$

и с учетом (22)

$$
\begin{aligned}
& \bar{x}_{0, k-1}(t)=(-1)^{k-1} a_{0}^{-}(t, 0) x^{(k-1)}(t)+O\left(x^{(k-2)}(t)\right) \\
& \tilde{x}_{0, k-1}(t)=(-1)^{i k-1} a_{0}^{+}(t, b) x^{(k-1)}(t-b)+O\left(x^{(k-2)}(t-b)\right) .
\end{aligned}
$$


Йтак,

$$
\begin{gathered}
u^{(k)}(t)=u(0) a_{0}^{-}(t, 0) x^{(k-1)}(t)-u(b) a_{0}^{+}(t, b) x^{(k-1)}(t-b)+z_{k}(t)+f^{(k)}(t)+ \\
+u(d)\left(\begin{array}{c}
k-1 \\
p
\end{array}\right)(-1)^{p}\left[\left.\frac{\partial^{p}}{\partial s^{p}} a_{0}^{+}(t, s)\right|_{s=d}-\right. \\
\left.\quad-\left.\frac{\partial^{p}}{\partial s^{p}} a_{0}^{-}(t, s)\right|_{s=d}\right] x^{(k-1-p)}(t-d),
\end{gathered}
$$

где $z_{k}(t)$ - некоторая непрерывная на $(0, d)$ и $(d, b)$ функция, такая, что

$$
\begin{gathered}
\left|z_{k}(t)\right| \leqslant c\left(t^{2(m-\beta)-k}+|t-d|^{2(m-\beta)-k+p}+(b-t)^{2(m-\beta)-k}+\left|x^{(k-2)}(t)\right|+\right. \\
\left.+\left|\boldsymbol{x}^{(k-2-p)}(t-d)\right|+\left|\boldsymbol{x}^{(k-2)}(t-b)\right|\right) .
\end{gathered}
$$

В силу неравенств (3) и (4) отсюда следуют предельные соотношения теоремы 2 при $v_{k}(t)=z_{k}(t)$. Таким же предельным соотношениям удовлетворяет $f^{(k)}(t)$, если $f \in C^{m}[0, b]$ или даже если $f \in E^{\alpha}$ с $\alpha<\gamma$, где $\gamma$ из (3). В результате приходим к представлению (5), (6), где $v_{k}(t)=z_{k}(t)+f^{(k)}(t)$. Для $k=1$ соответствующее представление следует из (8).

\section{Теорема 2 доказана.}

6. 3 а м е ч ан и е 1. Мы не предполагаем однозначной разрешимости уравнения (1). В случае $f=0$ теоремы 1 и 2 дают оценки производных собственных функций интегрального оператора в (1).

3 а м е ч н и е 2. Теоремы 1 и 2 справедливы и в случае, где $\beta$ целое число $(0<\beta<m)$. Тогда оценки (7) верны для всех производных, кроме производной порядка $k=m-1-\beta$, для нее из (2) вытекает

$$
\mid x^{(k)}(t) \leqslant c_{k}(|\ln | t||+1) .
$$

Соответственно, должно несколько отличаться поведение низших производных функции $u(t)$ в пространстве $E^{\beta}$.

3 а м е ч а и е 3 . Аналогичные теоремам 1 и 2 утверждения справедливы и в случае конечного числа $(N)$ линий разрывов по $s$ производных коэффициента $a(t, s)$, причем наименьшие числа $p_{i}(i=$ $=1, \ldots, N)$, при которых $\partial p_{t} a(t, s) / \partial s_{t}$ имеет при $s=d_{i}$ разрыв первого рода, могут быть различны $\left(0 \leqslant p_{i} \leqslant \beta\right)$.

3 а м е ч н и е 4. Если (3) имеет место с $\gamma=\beta$, то оценки производных решения интегрального уравнения (1), установленные теоремой 1 , точны по порядку.

\section{ЛИТЕРАТУРА}

1. Richter, G. R. J. Math. Anal. and Appl., 55, 32-42 (1976).

2. Schneider, C. Integr. Eq. Operator Theory, 2, 62-68 (1979).

3. Педас А. Уч. зап. Тартуск. ун-та, вып. 492, 56-68 (1979).

4. Вайникко $\Gamma$., Педас $A$. Оценки производных решения интегрального уравнения со слабо особым ядром. Тез. конференции «Теоретические и прикладные вопросы математики». Тарту, Изд-во Тартуск. ун-та, 1980, 193-195.

5. Vainikko, G., Pedas, A. J. Austral. Math. Soc., Ser. B, 22, 419-430 (1981).

6. Graham, I. G. J. Integr. Equat., 4, № 1, 1-30 (1982).

7. Уба П. Р. Приближенное решение интегрального уравнения со слабо особым ядром на неравномерной сетке. Канд. дис. Тарту, 1983.

8. Pitkäranta, J. SIAM J. Math. Anal., 11, 952-968 (1980).

9. Вайникко $Г$., Педас А., Уба П. Методы решения слабо-сингулярных интегральных уравнений. Тарту, 1984.

10. Владимиров В. С. Уравнения математической физнки. М., «Наука», 1971.

Тартуский государственный университет
Поступила в редакцию 4/V 1987 


\section{KATKEVA KORDAJAGA NORGALT SINGULAARSE INTEGRAALVORRANDI LAHENDI SILEDUS}

Vaadeldakse lineaarset II liiki integraalvõrrandit

$$
u(t)=\int_{0}^{b} a(t, s) x(t-s) u(s) d s+f(t), \quad 0 \leqslant t \leqslant b,
$$

kus vabaliige $f(t)$ on piisavalt sile $\left(f \in C^{m}[0, b], m \geqslant 1\right)$, aga funktsioonil $x^{(i)}(t)$ $(0 \leqslant i \leqslant m-1)$ on punktis $t=0$ integreeruv iseärasus. On teada, et $a(t, s) \in C^{m}([0, b] \times$ $\times[0, b])$ korral vōrrandi lahend $u(t)$ on vahemikus $(0, b) m$ korda pidevalt diferentseeruv, aga $u^{(k)}(t)$ käitub lõigu otspunktide lähedal nagu $x^{(k-1)}(t)$ nullpunktis $(i \leqslant k-1 \leqslant$ $\leqslant m-1)$.

Käesolevas artiklis on nõrgendatud tingimusi kordajale $a(t, s)$. Nimelt eeldatakse, et $p(0 \leqslant p \leqslant m)$ on vähim täisarv, mille korral funktsioonil $\frac{\partial^{p}}{\partial s^{p}} a(t, s)$ on sirgel $s=d$ esimest liiki katkevus. Selgub, et sellisel juhul käitub võrrandi lahend $u(t) \in C^{p}(0, b) \cap$ $\cap C^{m}((0, b) \backslash\{d\})$ löigu otspunktide ümbruses nagu varem, aga punkti $t=d$ ümbruses käitub funktsioon $u^{(p+l)}(t) \quad(1 \leqslant l \leqslant m-p)$ nagu funktsioon $x^{(l-1)}(t)$ nullpunktis.

\section{P. UBA}

\section{THE SMOOTHNESS OF SOLUTION OF WEAKLY SINGULAR INTEGRAL EQUATION WITH A DISCONTINUOUS COEFFICIENT}

We examine the differential properties of the solution of the linear integral equation

$$
u(t)=\int_{0}^{b} a(t, s) x(t-s) u(s) d s+f(t), \quad 0 \leqslant t \leqslant b,
$$

where $x \in C^{m-1}([-b, b] \backslash\{0\}), m \geqslant 1$ and, for $-b \leqslant t<0$ and $0<t \leqslant b, \mid x^{(m-1)}(t) \leqslant$ $\leqslant c|t|^{-\beta}$ is valid with $0<\beta<m$.

It is well known (see $\left.\left[{ }^{1-9}\right]\right)$ that in case of $f \in C^{m}[0, b]$ and $a(t, s) \in C^{m}([0, b] \times$ $\times[0, b])$ a singularity of $x^{(k-1)}(t) \quad(1 \leqslant k \leqslant m)$ at 0 determines the singularities of the same order of $u^{(k)}(t)$ at 0 and $b$.

In this paper we study a case with weaker conditions on $a(t, s)$. We assume that $p$ is the least integer, by which the function $\frac{\partial^{p}}{\partial s^{p}} a(t, s)$ has the first kind discontinuity on the straight line $s=d(0<d<b)$. We prove that, in addition to singularities at the end points, the $(p+l)$-th derivative $(1 \leqslant l \leqslant m-p)$ of the solution $u(t)$ behave at the point $d$ as the function $x^{(l-1)}(t)$ at 0 . 